\title{
Criminologie
}

\section{Fratricide et sororicide : synthèse de la littérature}

\author{
Jacques D. Marleau
}

Volume 36, numéro 1, printemps 2003

Police et prévention : évaluation et analyse d'impact

URI : https://id.erudit.org/iderudit/006558ar

DOI : https://doi.org/10.7202/006558ar

Aller au sommaire du numéro

Éditeur(s)

Les Presses de l'Université de Montréal

ISSN

0316-0041 (imprimé)

1492-1367 (numérique)

Découvrir la revue

Citer cet article

Marleau, J. D. (2003). Fratricide et sororicide : synthèse de la littérature.

Criminologie, 36(1), 157-175. https://doi.org/10.7202/006558ar

\section{Résumé de l'article}

Cette synthèse trace le bilan des recherches cliniques et épidémiologiques des écrits sur le fratricide et le sororicide et présente les facteurs situationnels, dynamiques et psychopathologiques, ainsi que les motivations liées au geste. Une recherche bibliographique effectuée à l'aide de plusieurs bases de données a permis d'identifier et de synthétiser 43 textes sur le sujet. Trois profils types d'agresseurs selon l'âge lors du passage à l'acte ont été identifiés, soit les enfants âgés de moins de 11 ans, les pré-adolescents/adolescents, âgés de 11 à 17 ans, et les adultes âgés de 18 ans et plus. Les facteurs associés au passage à l'acte varient selon les trois groupes d'agresseurs. Le rôle des parents est important dans la prévention des passages à l'acte violent chez les enfants et les adolescents. Des recommandations pour les recherches futures sont aussi présentées.
Ce document est protégé par la loi sur le droit d'auteur. L'utilisation des services d'Érudit (y compris la reproduction) est assujettie à sa politique d'utilisation que vous pouvez consulter en ligne.

https://apropos.erudit.org/fr/usagers/politique-dutilisation/ 


\title{
Fratricide et sororicide: synthèse de la littérature
}

\author{
Jacques D. Marleau \\ Centre de recherche de l'Institut Philippe Pinel \\ Montréal \\ marleauj@ videotron.ca
}

\begin{abstract}
RÉSUMÉ - Cette synthèse trace le bilan des recherches cliniques et épidémiologiques des écrits sur le fratricide et le sororicide et présente les facteurs situationnels, dynamiques et psychopathologiques, ainsi que les motivations liées au geste. Une recherche bibliographique effectuée à l'aide de plusieurs bases de données a permis d'identifier et de synthétiser 43 textes sur le sujet. Trois profils types d'agresseurs selon l'âge lors du passage à l'acte ont été identifiés, soit les enfants âgés de moins de 11 ans, les préadolescents/adolescents, âgés de 11 à 17 ans, et les adultes âgés de 18 ans et plus. Les facteurs associés au passage à l'acte varient selon les trois groupes d'agresseurs. Le rôle des parents est important dans la prévention des passages à l'acte violent chez les enfants et les adolescents. Des recommandations pour les recherches futures sont aussi présentées.
\end{abstract}

ABSTRACT - This paper aims to describe clinical and epidemiological factors about fratricide and sororicide. It is based on situational, dynamic and psychopathological assessment, and to act-related motivations. A bibliographic search on many databases allowed the identification and synthesis of 43 papers on the matter. Three profiles of agressors, according to age, were identified: children under 11, pre-teenagers/teenagers from 11 to 17, and the adults. The parental role is important in prevention of acting-outs of children and teenagers. Further recommendations for future research are also included.

\section{Introduction}

Les homicides intrafamiliaux les plus fréquents sont l'uxoricide (homicide de la conjointe), le parricide (homicide du père), le filicide (homicide d'un enfant par un ou les deux parents) et le matricide (homicide de la mère). Plusieurs écrits ont été publiés sur chaque type d'homicide au cours 
des dernières années (M illaud et al., 1996 ; Ewing, 1997; Boisvert et Cusson, 1999 ; H illbrand et al., 1999 ; M arleau et al., 1995; 1999); nous savons cependant peu de choses sur les homicides entre frères et/ ou sœurs.

Ce manque d'informations étonne, puisque la violence entre frères et/ ou sœurs est une des formes de violence les plus importantes à l'intérieur des familles (G elles et Cornell, 1990). Au cours de l'enfance et de l'adolescence, la violence est plus souvent présente quand les individus sont du même sexe, ou encore lorsque l'écart entre les naissances est mince (Leung et Robson, 1991). Les données américaines de Strauss et al. (1980, dans G elles et Cornell, 1990) indiquent que $82 \%$ des enfants âgés de 3 à 17 ans ont eu recours à la violence physique envers un ou plusieurs membres de leur fratrie au cours de la dernière année. Plusieurs parents et professeurs banalisent ces agirs et considèrent même qu'ils fournissent des balises aux enfants afin de contrôler leur agressivité dans de futures situations concrètes.

La rivalité entre l'agresseur et la victime est I'hypothèse la plus souvent avancée pour comprendre les homicides entre frères et/ ou sœurs. Cette explication s'inspire en partie de la théorie psychodynamique où les enfants pourraient avoir le souhait inconscient de tuer des membres de leur famille (Sulloway, 1996). D'autres considèrent que les agressions représentent un déplacement de l'hostilité parentale vers un membre de la fratrie (D uncan et D uncan, 1971). M ais qu'en est-il vraiment dans la réalité? Cet article vise à faire la synthèse des écrits, autant cliniques qu'épidémiologiques, sur le fratricide et le sororicide, afin de donner un portrait des agresseurs et des victimes impliqués, ainsi qu'un aperçu des facteurs situationnels, dynamiques et mentaux qui y sont associés.

\section{Méthodologie}

Les textes cités dans cet article ont été trouvés à l'aide des bases de données M edline (1966 à 2002), Psyclnfo (1967 à 2002), N ational C riminal Justice Service Abstracts (1968 à 2002), Sociological Abstracts (1986 à 2002) et Social Science Index (1983 à 2002). Les mots-clés nécessaires à l'identification des manuscrits étaient fratricide, sororicide, homicide, meurtre («murder»), frères («brothers»), sœurs («sisters») et fratrie («sibling[s]»). D'autres textes ont été trouvés en consultant la bibliographie des articles identifiés. Les textes retenus devai ent avoir été publiés entre 1960 et 2002. Q uarante-trois documents traitant du phénomène du fratricide/ sororicide ont donc été identifiés. 


\section{Définitions}

Le mot fratricide est le terme le plus souvent employé dans la littérature sur les homicides entre frères et/ ou sœurs. Le dictionnaire L e Petit Robert (1987) donne deux définitions. La première se réfère à l'action de tuer un frère ou une sœur. La racine du mot provient du bas latin fratricidium. M ahieu (1998) explique que cette expression latine est composée de frater (frère) et de caedere (tuer). La seconde désigne la personne qui tue son frère ou sa sœur.

Au cours des dernières années, certains auteurs ont employé le terme «sororicide» (Adam et Livingston, 1993; Ewing, 1990b; 1997) pour désigner spécifiquement I'homicide d'une sœur. Ce mot est surtout employé dans la littérature anglophone.

D'autres font plutôt référence à l'expression «complexe de Caïn » («C ain Complex »; Sulloway, 1996) en référence au geste fatal posé par Caïn envers son frère Abel. Rabain (1995) avance l'idée de tentation fratricide que les individus doivent surmonter au cours de l'enfance et de l'adolescence. Underwood et Patch (1999) emploient le terme «siblicide» pour désigner les homicides entre frères et/ ou sœurs (voir aussi Daly et al., 2001; G ebo, 2002). Ce mot est aussi fréquemment employé dans les recherches sur les oiseaux (M ock, 1984 ; Forbes et M ock, 1994), les poissons (Dominey et Blumer, 1984) et les invertébrés (Polis, 1984). Aucune équivalence en français n'existe cependant.

Pour terminer, mentionnons que le terme fratricide peut également signifier l'action meurtrière entre hommes et/ ou femmes qui font partie d'un groupe comme une fraternité. $0 \mathrm{n}$ retrouve régulièrement des expressions du type guerre fratricide (par exemple au Rwanda, au Sri Lanka et en Algérie) dans les communiqués de presse. Coy et Steinweg (1996) parlent aussi de fratricide, mais entre soldats du même camp. Ils donnent trois exemples de militaires qui ont abattu des camarades lors d'un conflit armé.

\section{Historique}

L'histoire de l'humanité est jalonnée de meurtres entre membres de la même fratrie. Le premier cas est celui de Caïn et Abel. Q ui ne connaît pas cet événement, qui relate que Dieu préféra I'offrande d'Abel et que Caïn, irrité par ce geste, tua son frère (Bible, Genèse 4 : 3-8). Reouven (1986) note une histoire similaire dans la mythologie polynésienne: Tribo, un des fils de Tangaroa, le Dieu des pêcheurs, tue par jalousie son frère 
Yaka-A ko-O uli. D'autres cas célèbres ont également été retracés dans la Bible; on n'a qu'à penser à Abimélech, égorgeant ses frères et demifrères aînés afin d'hériter de son père et d'être roi (Bible, Juges $9: 5$ ) ou à Absalon, ordonnant à ses serviteurs de tuer son demi-frère Amnon qui avait violé Thamar, sa sœur (Bible, II Samuel 13 : 28-29). D eux exemples de rivalité fraternelle sont aussi à souligner : le premier concerne Jacob qui a réussi à obtenir le droit d'aînesse de son frère Ésaü en l'échangeant contre un plat de lentilles (Bible, Genèse 25 : 29-34); le second est celui de Joseph (fils de Jacob) vendu par ses frères aînés à des marchands Ismaélites, car il était le favori de leur père (Bible, Genèse 37 : 25-28; Leung et Robson, 1991).

Plusieurs histoires de violence fraternelle reliée à une question d'héritage ou à une prise de pouvoir ont également été identifiées au cours de I'H istoire. On n'a qu'à penser à D ominicien, César Borgia, R ichard III, la femme du comte Hyppolite de Bocarmé, les fils d'Henri VIII et le sultan M ehemmed III (Reouven, 1986; Sulloway, 1996). D aly et Wilson (1988) mentionnent également que l'histoire des familles royales est marquée de plusieurs meurtres entre les membres de la même fratrie, très souvent pour la succession. Au Danemark, au xvi siècle, les querelles entre frères au sujet de la succession étaient tellement nombreuses qu'une loi avait été adoptée pour empêcher qu'un fratricide puisse hériter.

D 'autres cas célèbres portent plutôt sur la rival ité en association avec un ou des éléments de frustration présents entre deux frères. L'histoire de Romulus et Remus est bien connue. Romulus avait été désigné pour construire l'enceinte de la ville de Rome. Déçu de ne pas avoir été sélectionné, Remus enjamba d'un bond l'enceinte lorsqu'elle fut terminée. 0 utragé par ce geste, Romulus tua son frère (Reouven, 1986). Un autre exemple est celui de l'affrontement entre les Horaces et les Curiaces, représentant respectivement la ville de Rome et d'Albe. Horace tua sa sœur qui avait comme amant un des Curiace (M ahieu, 1998). Un autre exemple est celui des frères $D$ iaz, qui se produisit au milieu du xvi siècle. Le frère aîné Alfonso, catholique, qui était avocat à la cour papale de Rome, fit assassiner son jeune frère Juan, à la suite de sa conversion au protestantisme (Sulloway, 1996).

Les mythes et légendes sont un répertoire sans fin de meurtres intrafamiliaux. Les homicides entre frères et/ ou sœurs ne font pas exception à cette règle. Les exemples les plus notoires sont ceux de Seth et $d^{\prime} O$ siris, et d'Étéocle et de Polynice. Ce dernier drame se termine par un double fratricide. Soulignons égal ement le cas de $M$ édée. C ette dernière 
est connue surtout pour avoir tué les deux garçons issus de son union avec Jason (syndrome de M édée), mais on oublie trop souvent qu'elle avait auparavant assassiné son frère Alsyrtos.

\section{Prévalence}

Daly et Wilson (1988) ont présenté, dans leur livre H omicide, des données sur les fratricides dans les sociétés agricoles. IIs représentent entre 7 et $10 \%$ des homicides, un nombre élevé, mais allant dans le sens de leur prédiction, à savoir que dans ce type de société la succession est habituellement transmise à l'aîné, ce qui peut facilement engendrer des conflits avec les autres frères. Ils n'observent pas de cas dans les sociétés de chasseurs-cueilleurs, la raison la plus plausible étant l'absence de propriété à transmettre.

Les données sur les homicides entre frères et/ ou sœurs sont rares dans les sociétés contemporaines. Celles compilées à partir d'informations sur les villes de Philadelphie (de 1948 à 1952) et de N ew York (en 1965), aux États-Unis, montrent que les meurtres entre frères et/ ou sœurs représentent $3 \%$ des homicides (Gelles et Cornell, 1990). Les résultats de Daly et Wilson (1988) pour la ville de Detroit, en 1972, indiquent que 1,4\% des homicides sont des fratricides (7/ 508). Des données plus récentes, recueillies par le Département américain de la justice, indiquent que 1,5\% des homicides sont des fratricides/ sororicides (Ewing, 1997). Au Canada, le pourcentage d'homicides entre frères et/ ou sœurs représente 2,1\% des homicides résolus en 1998 (Fedorowycz, 1999). En Australie, Wallace (1986) présente des données sur les homicides intrafamiliaux de la province de N ouvelle-Galles du Sud; les meurtres entre frères et/ ou sœurs représentent $4,2 \%$ des homicides, un pourcentage supérieur à celui des États-U nis et du Canada.

Certains auteurs comme Cormier et al. (1978) estiment que la prévalence des fratricides est sous-évaluée. Selon eux, certains parents protégeraient leurs jeunes enfants du système judiciaire en camouflant l'incident aux autorités; le décès serait alors considéré comme accidentel.

\section{Le profil des agresseurs}

Les fratricides sont majoritairement des hommes. Aux États-Unis, les pourcentages oscillent entre $73 \%$ et $88 \%$ (Ewing, 1997 ; Underwood et Patch, 1999 ; Daly et al., 2001 ; Gebo, 2002). On retrouve des pour- 
centages similaires au Canada (M arleau et Saucier, 1998) et en Australie (Wallace, 1986).

Aux États-Unis, I'âge moyen des agresseurs est de 34,4 ans (Underwood et Patch, 1999). La majorité ont 18 ans et plus (87\%) au moment du passage à l'acte (voir aussi Ewing, 1997). Les données australiennes de Wallace (1986) vont dans le même sens. Au Canada, $56 \%$ des agresseurs sont âgés de 20 ans et plus (M arleau et Saucier, 1998). II est à noter, toutefois, que le groupe d'âge comparé n'est pas le même (18 ans et plus vs 20 ans et plus), ce qui peut expliquer une partie des variations constatées.

Les résultats de M arleau et Saucier (1998) et de Wallace (1986) indiquent que les agresseurs sont en général plus jeunes que les victimes. Ces résultats vont à l'encontre de la prédiction de Sulloway (1996), selon laquelle les agresseurs seraient plus âgés. Cependant, D aly et al. (2001) démontrent qu'ils sont plus souvent âgés lorsque les deux protagonistes sont âgés de 14 ans ou moins au Canada (1974-1990), en Angleterre (1977-1990) et dans la ville de C hicago, aux États-U nis (1965-1994).

Aux États-U nis, les passages à l'acte sont plus nombreux dans les régions du Sud. La majorité des passages à l'acte sont commis par des gens de race blanche ou noire (Ewing, 1997 ; Underwood et Patch, 1999). Toutefois, lorsque comparé à leur poids relatif dans la population générale, le taux est plus élevé chez les Hispano-Américains et moins élevé chez les Asiatiques (Underwood et Patch, 1999). Aucune donnée n'a été présentée sur le Canada et l'Australie selon un découpage géographique ou l'origine ethnique des agresseurs.

Les données américaines indiquent qu'au moment du passage à l'acte environ $33 \%$ des agresseurs étaient sans emploi et $71 \%$ avaient un dossier criminel (Ewing, 1997). On ne retrouve aucune information sur l'état civil ou le niveau de scolarité des agresseurs à partir des études épidémiologiques. Les informations concernant la consommation de psychotropes indiquent que la moitié des agresseurs avaient consommé de l'alcool au moment du passage à l'acte (Ewing, 1997). Les données de Underwood et Patch (1999) révèlent des taux plus bas; ils font I'hypothèse d'une sous-estimation de la consommation de psychotropes. Wallace (1986) mentionne que la consommation d'alcool est reliée au passage à l'acte dans la majorité des cas où des adultes sont impliqués. 


\section{Le profil des victimes}

Les victimes sont le plus souvent des hommes. Plusieurs auteurs confirment que la combinaison agresseur-victime frère-frère est la plus souvent rencontrée (Ewing, 1997; M arleau et Saucier, 1998; Underwood et Patch, 1999 ; D aly et al., 2001). La combinaison sœur-sœur est très rare et varie entre 4 et $12 \%$ (Daly et al., 2001).

\section{Caractérisation des délits}

Peu d'informations sont disponibles sur le lieu de l'agression. À notre connaissance, Wallace (1986) est l'un des seuls à mentionner que, lors du passage à l'acte, l'agresseur et la victime vivent souvent ensemble (voir aussi Ewing, 1997). L'examen des rapports de cas identifiés dans la littérature montre que, pour les cas où l'information est disponible, la majorité d'entre eux vivaient ensemble lors du passage à l'acte.

Le moyen utilisé lors du passage à l'acte est souvent mentionné dans les écrits. Les agresseurs de l'échantillon d'Ewing (1997) ont utilisé une arme à feu dans $40 \%$ des cas. Underwood et Patch (1999) observent que la majorité des agresseurs ont utilisé une arme à feu ou un couteau/ objet contondant. Ces données proviennent de recherches effectuées aux ÉtatsUnis. Les moyens utilisés dans d'autres pays pourraient différer, compte tenu des législations propres à chaque pays sur les armes à feu. Les données sur les rapports de cas identifiés révèlent aussi une utilisation importante des armes à feu, suivi du couteau/ objet contondant et de l'utilisation des mains (strangulation, coups). II est important de souligner que la très grande majorité des cas proviennent égal ement des États-Unis et que cela explique probablement la prépondérance des armes à feu.

\section{Facteurs situationnels}

Ce type de facteurs fait référence aux facteurs de stress psychosociaux survenus au cours de l'année précédant le délit et à ceux associés à l'acte violent. Ewing (1997) note plusieurs facteurs importants de stress, comme une séparation des parents, le chômage et une consommation de psychotropes, autant chez les adolescents que chez les adultes. Le fait de vivre avec un membre de sa famille, surtout parmi les sujets adultes, se surajoute aux stresseurs psychosociaux; pour plusieurs, cette situation constitue une situation anxiogène difficile à vivre. II constate aussi, chez certains sujets, 
une dépendance financière à leur frère et/ ou sœur (phénomène rencontré aussi chez certains parricides). Plusieurs mentionnent que les parents de certains adolescents agresseurs se sont séparés peu de temps avant le délit (M archand et Basse, 1986; A dam et Livington, 1993). D ans certains cas, la reconstitution de la famille avec de nouveaux acteurs (belle-mère, demi-frère, etc.) semble avoir contribué au passage à l'acte, surtout durant I'adol escence. Dans d'autres cas, le passage à l'acte a été commis sous l'influence de l'alcool (Briguet-Lamarre, 1969; Wallace, 1986). Certains mentionnent des facteurs précis tels que les problèmes de santé du père (Woods, 1961) ou le refus du père à plusieurs demandes (Gardiner, 1985) comme éléments associés au passage à l'acte.

Ewing (1990a, 1997) rapporte que des variables comme l'abus physique et la négligence parentale contribuent au passage à l'acte dans certaines situations, surtout lorsque les agresseurs et les victimes sont des enfants et/ ou des adolescents (voir aussi Tooley, 1975). Cependant, l'auteur n'explique pas le lien entre l'abus et/ ou la négligence parentale et le passage à l'acte violent. II est possible que la présence de modèles violents contribue à l'apprentissage de réactions violentes lors de situations conflictuelles/ stressantes. D es cas de violence physique entre membres de la fratrie sont également à souligner parmi les adolescents (Woods, 1961 ; Leong, 1989).

\section{Facteurs psychologiques}

Un des facteurs mentionnés le plus souvent est celui de la rivalité entre les frères et/ ou les sœurs, que l'on retrouve dans plusieurs histoires de cas. Patterson (1943) insiste sur ce point et mentionne que les attaques sadiques sont nombreuses entre membres de la fratrie. Ewing (1990b; 1997) ajoute que cette explication seule est trop réductionniste, voire simpliste. M algré cette réserve, on constate souvent que les relations entre la victime et l'agresseur sont teintées d'ambivalence, soit d'amour et de haine, surtout parmi les enfants et les adolescents. Certains suggèrent la présence de conflits non résolus associés au processus de compétition pour l'attention parentale (Wallace, 1986). D'autres mentionnent que la naissance (M cCully, 1978) ou l'adoption (M ahieu, 1998) de la future victime a été perçue par l'agresseur comme un rejet de la part des parents, surtout de la mère.

D ans certains cas identifiés dans la littérature, l'agresseur percevait que l'attention d'un ou des parents était centralisée sur d'autres membres de la fratrie. Le passage à l'acte s'inscrirait donc dans une logique d'obtention de l'affection des parents, une dimension de jalousie étant souvent 
présente (Schmideberg, 1973 ; Paluszny et M cN abb, 1975 ; Scherrer, 1980), surtout parmi les adolescents. D'autres suggèrent que le passage à I'acte vise à faire souffrir un ou les deux parents (Gardiner, 1985; M archand et Basse, 1986 ; Ewing, 1990b ; Adam et Livingston, 1993). Russell (1986) mentionne que, dans certains cas, le geste est la réaction à des conflits parentaux profonds. Les désirs incestueux (Woods, 1961) et/ ou I'hostilité/ agressivité (Russell, 1986; Ewing, 1997) auraient été déplacés de la mère à un membre de la fratrie. Duncan et $D$ uncan (1971) croient également que la victime représente souvent un parent haï. Plusieurs ont des fantasmes familicides avant le passage à l'acte (Woods, $1961 ; 0$ chonisky, 1963; Russell, 1986). La réalisation de ce fantasme leur permettrait de faire les choses qu'ils désirent, comme par exemple vivre seul avec leur père (Woods, 1961) ou fréquenter le conjoint qu'elle aime (0 chonosky, 1963). Certains mentionnent que les mères ont une personnalité dominante et qu'elles ont souvent fait des reproches à I'agresseur (Woods, 1961; Russell, 1986; William, 1988; Bornstein et al., 1995), tandis que le père est carrément absent physiquement et/ ou émotivement. D'autres, comme Tooley (1975), suggèrent que certains enfants ont agi pour aider leur mère. Cette dynamique est aussi présente chez certains parricides (voir Sargent, 1962).

II y a aussi des cas où les parents encouragent les conflits, ou du moins ne réussissent pas à empêcher la victime de frapper et/ ou de dénigrer I'agresseur (Adam et Livingston, 1993). Ce type de situation sert de toile de fond au passage à l'acte. Lorsque l'agresseur est plus jeune que la victime, il est souvent sous le joug punitif/ autoritaire ou défavorisé par rapport à l'aîné (Briguet-Lamarre, 1969 ; Russell, 1986 ; Wallace, 1986). L'inverse est aussi constaté, c'est-à-dire que l'agresseur est plus âgé que la victime. Ce genre de situation survient souvent en l'absence des parents lorsque l'aîné garde les plus jeunes et que ceux-ci contestent son autorité (Carek et Watson, 1964 ; William, 1988). II arrive que la future victime ait menacé l'agresseur de rapporter ce qui s'était passé (Russell, 1986; William, 1988) ou de dire un mensonge (Gardiner, 1985) aux parents. Plusieurs insistent sur l'absence des parents du domicile familial comme élément prédisposant, autant chez les enfants (Adelson, 1972; Paluszny et $M$ cN abb, 1975 ; Petit et Wells, 1980 ; Adam et Livingston, 1993) que chez les adolescents (M archand et Basse, 1986; Russell, 1986).

II est important de mentionner que plusieurs enfants ne voulaient pas tuer leur frère et/ ou leur sœur, mais que le geste a été impulsif (Tooley, 1975 ; Ewing, 1990a). Wallace (1986) mentionne qu'il y a rarement 
préméditation, ou même intention, chez les fratricides adultes comparativement à d'autres types de délit (voir aussi Duncan et D uncan, 1971). Chez les jeunes agresseurs, la thèse de l'accident est validée en partie par le fait que la notion de mort est pour eux difficile à cerner et à comprendre (Tooley, 1975). Selon Duncan et D uncan (1971), ils perçoivent les individus comme des irritants plutôt que des êtres humains. Plusieurs ne perçoivent pas l'irréversibilité de leur geste et ne réalisent pas que la mort est la conséquence de leur action. La situation ayant précipité le geste est souvent anodine, dispute pour la télévision (William, 1988), remarque désobligeante du frère (Briguet-Lamarre, 1969 ; Stone, 1993), dispute pour un vélo (Ewing, 1997), cris du plus jeune (Adelson, 1972; Paluszny et $\mathrm{M} c \mathrm{~N}$ abb, 1975), disputes concernant de l'argent et/ ou de la drogue (D ecker, 1996) et taquinerie de la part de la victime (Petit et Wells, 1980).

L'examen des rapports de cas indique que plusieurs des victimes sont des demi-frères ou des demi-sœurs (Foster, 1964 ; M cCully, 1978; M archand et Basse, 1986). La séparation semble avoir joué un rôle important chez certains adolescents en modifiant la dynamique des relations existantes et en ajoutant de nouveaux acteurs dans la famille. Par exemple, M archand et Basse (1986) soulignent que la mésentente entre l'agresseur et la bellemère n'est pas étrangère au passage à l'acte de l'adolescent. Wallace (1986) émet I'hypothèse qu'il y aurait plus d'antagonisme et de rivalité entre les demi-frères/ demi-sœurs (voir Daly et al., 2001). Kashani et al. (1997) insistent plutôt sur l'adoption comme étant un élément négatif important (voir aussi Leong, 1989; Mahieu, 1998). Le cas présenté par M ahieu (1998) montre l'importance de bien préparer l'enfant aîné à la venue d'un nouveau-né. M archand et Basse (1986) décrivent le cas d'un adolescent ayant un problème possible d'identité familiale (pris entre deux familles). M ahieu (1998) parle plutôt d'un problème d'identité culturelle. D'autres mentionnent un problème d'identité sexuelle (Russell, 1986). Dans quelques cas, on peut même parler d'un problème d'identité gémellaire (Petit et Wells, 1980 ; M ahieu, 1998). La recherche d'une identité propre, lorsqu'elle est court-circuitée, constitue un élément associé au passage à l'acte, surtout lors de la préadolescence et del'adolescence (Russell, 1986).

Plusieurs autres facteurs, tels que l'agressivité de l'agresseur et le concept de déshumanisation, peuvent aussi avoir joué un rôle important. D'autres parlent de l'immaturité et du peu de contrôle de l'expression des émotions et des impulsions chez plusieurs enfants et adolescents agresseurs (M iller et Looney, 1974 ; K ashani et al., 1997). 


\section{Facteurs associés à la santé mentale}

L'examen du profil de santé mentale des enfants et des adolescents montre qu'ils souffrent rarement de psychose au moment du passage à I'acte. Ceux qui présentent des éléments psychotiques ont effectué le passage à l'acte lors d'un épisode délirant (Petit et Wells, 1980; M archand et Basse, 1986) ou en lien avec des hallucinations auditives mandatoires où le Diable est une figure importante (M cCully, 1978; Benedek et Cornell, 1989). D ans plusieurs situations fratricides, les adolescents souffraient de troubles des conduites (Ewing, 1997).

En ce qui concerne les agresseurs d'âge adulte, peu d'informations sont disponibles (Woytessek et Atwal, 1985; Roman et Annis, 1986; Stone, 1993 ; Ewing, 1997). Roman et Annis (1986) rapportent le cas d'une psychiatre assassinée par son frère schizophrène. L'agresseur avait des hallucinations auditives lui ordonnant de tuer sa sœur. Ce concept du «moi ou l'autre» est souvent rencontré chez les parricides psychotiques (voir Zagury et M illaud, 1998). Stone (1993) raconte le cas d'un homme de 33 ans qui a tué 11 personnes (son frère, sa femme, leurs 8 enfants et sa mère). L'agresseur, un schizophrène, avait depuis plusieurs années des idées délirantes de persécution où son frère jouait le rôle de persécuteur. Woytassek et Atwal (1985) présentent le cas d'un homme atteint du syndrome de Capgras (maladie du double) qui a tué sa sœur. Depuis plusieurs années, il croyait que des membres de sa famille avaient été remplacés par des imposteurs. Le dernier cas est celui d'un homme avec un passé psychiatrique lourd qui a tué sa sœur enceinte parce qu'il croyait que l'enfant serait malade (Ewing, 1997).

\section{Classification des délits}

Comparativement aux auteurs ayant étudié d'autres types de meurtres intrafamiliaux tels que le filicide ou le parricide, les auteurs qui se sont attardés au fratricide/ sororicide n'ont que rarement tenté de classifier les passages à l'acte entre frères et/ ou sœurs. M ahieu (1998) a récemment tenté de les classer en trois grandes catégories. La première fait référence à la notion de séparation. Le passage à l'acte serait une stratégie visant à produire une séparation d'avec les frères et/ ou sœurs; cela ressemble à la notion du «moi ou l'autre» mentionnée ci-dessus. La seconde est associée à l'inceste. M ahieu (1998) va jusqu'à sous-entendre le déplacement d'imagos parentales sur un frère ou une sœur. Q uelques cas sont rapportés dans la littérature: Ewing, 1997 (meurtre du frère et viol de la sœur); Sherrer, 
1980 (viol et meurtre de la sœur) et Woods, 1961 (tentative de viol, puis meurtre de la so eur). La troisième s'inscrit dans la dialectique de l'avoir (de la possession); ce qui est important est la possession de l'objet de convoitise. Plusieurs fratricides/ sororicides font référence à cette dernière catégorie où l'agresseur et la victime convoitent une personne et/ ou un objet.

Sadoff (1995) a décrit quelques situations types rencontrées en clinique où le motif le plus probant est la rivalité entre les deux acteurs. Chez plusieurs, il constate la présence d'une altercation dans un contexte où l'un ou les deux individus étaient intoxiqués. II mentionne égal ement qu'il est très rare de rencontrer des cas où la vengeance est le moteur principal de l'action (pour certains cas, voir Gardiner, 1985 ; M archand et Basse, 1986) ou des cas où la pathologie du sujet joue un rôle important (psychose). Enfin, plusieurs adolescents agresseurs souffrent de troubles des conduites, ce qui, selon lui, peut faciliter le passage à l'acte.

Comme nous l'avons mentionné plus haut, certains cas peuvent être considérés comme des accidents (Sturner et al., 1991; Ewing, 1997), surtout chez les jeunes agresseurs. Certains auteurs laissent sous-entendre que, malgré les apparences d'un accident, l'intention de tuer pouvait être présente chez certains sujets (Russell, 1986).

$0 \mathrm{n}$ ne retrouve que très rarement des motivations à caractère altruiste chez les fratricides, comparativement aux filicides par exemple. Nous avons recensé uniquement un cas où la dimension altruiste était présente (Leong, 1989). La dimension suicidaire souvent présente dans les homicides altruistes était absente lors du passage à l'acte. M edlicott (1970) se demande toutefois si le passage à l'acte fratricide de l'agresseur ne représente pas symboliquement la destruction d'une partie de lui-même.

D ans certaines situations, l'agresseur s'en prend à plusieurs personnes, habituellement des membres de sa famille, ce qui suggère la présence d'une étiologie différente. Les frères et/ ou les sœurs sont souvent des victimes secondaires. Par exemple, Gardiner (1985) rapporte le cas d'un adolescent qui a tué sa mère, puis ses deux sœurs, dans un moment de panique. $D$ 'autres ont été tués ou blessés en voulant défendre leurs parents, tandis que d'autres encore ont été tués car ils faisaient partie de l'ambiance négative du milieu familial.

\section{Aspect pénal et considération de traitement}

À notre connaissance, aucune donnée épidémiologique n'a été publiée sur les aspects juridiques (accusations, sentences, etc.). II est possible de trou- 
ver certaines informations dans les rapports de cas identifiés dans la littérature. Cependant, il est difficile d'en tirer des conclusions définitives, compte tenu des nombreuses époques ciblées et aussi de l'orientation des auteurs variant selon leur discipline d'intérêt. Q uelques-uns rapportent le traitement clinique d'enfants et d'adol escents (Carek et Watson, 1964; Paluszny et $\mathrm{M}$ acN abb, 1975 ; Petit et Wells, 1980 ; Williams, 1988) ou la réaction négative des membres du personnel traitant à la suite du passage à l'acte (M archand et Basse, 1986 ; Williams, 1988).

\section{Discussion}

Cette synthèse nous a permis de constater qu'il existe beaucoup d'informations sur les meurtres entre frères et/ ou sœurs dans la littérature nord-américaine (surtout américaine) des 40 dernières années. Nous avons dégagé trois portraits types selon l'âge des agresseurs: les enfants âgés de moins de 11 ans, les pré-adolescents/ adolescents âgés de 11 à 17 ans et finalement les adultes, âgés de 18 ans et plus.

Le premier groupe est constitué des enfants, pour qui la notion de mort est un concept flou. Leur geste est souvent posé de façon spontanée lors d'une altercation pour obtenir un objet ou lors d'une situation de frustration (pleurs d'un bébé, par exemple). La situation type est celle où l'aîné a la responsabilité de ses frères et/ ou sœurs et que l'un d'eux défie son autorité (ne veut pas aller dans sa chambre, par exemple). II se produit souvent une altercation physique où le plus jeune est tué accidentellement. $0 \mathrm{n}$ constate également que la disponibilité d'armes blanches et/ ou d'armes à feu (surtout aux États-U nis) est un élément important. L'absence des parents est un élément majeur, mais dans certains cas on peut aussi parler de pratiques éducatives déficientes, c'est-à-dire que certains agresseurs ont de la difficulté à contrôler leur impulsivitél agressivité et n'ont pas appris à négocier dans certaines situations de frustration. Chez plusieurs, on remarque un passé d'abus physiques et/ ou sexuels de la part d'un des parents, pouvant favoriser l'utilisation de la violence dans certaines situations. $D$ 'autres notent que des parents favorisent certains membres de la fratrie au détriment d'autres (habituellement l'agresseur), pouvant ainsi amplifier la rivalité entre les membres de la fratrie.

On retrouve certains éléments mentionnés ci-dessus dans le portrait des adolescents agresseurs. Les plus significatifs sont également les abus physiques et/ ou sexuels rencontrés chez certains. Les notions d'impulsivité, d'immaturité et de déshumanisation sont souvent présentes. 
Plusieurs souffrent de troubles des conduites lors du passage à l'acte (à mettre en lien avec la notion de déshumanisation). C'est dans ce groupe qu'on retrouve le plus d'exemples où l'intention de tuer était présente et de familicides (c'est-à-dire que les parents et/ ou les grands-parents sont aussi des victimes). O n constate aussi que les parents utilisent souvent des pratiques éducatives inappropriées en favorisant un ou des membres de la fratrie ou en complimentant certains plutôt que d'autres. Certains agresseurs sont clairement identifiés comme les «moutons noirs». Des passages à l'acte sont d'ailleurs commis dans un contexte de rivalité intense, surajoutée à un élément de vengeance à l'égard de la victime ou d'un parent (souvent celui qui favorise la victime). Enfin, c'est dans cette catégorie d'âge que l'on constate le plus de meurtres à l'égard de demifrères/ demi-sœurs ou d'enfants des nouveaux conjoints, suggérant des difficultés d'adaptation marquées à cette nouvelle situation.

Le groupe des adultes se divise en deux catégories. La première catégorie regroupe les agresseurs effectuant un passage à l'acte lors d'une altercation sur des thèmes comme l'argent et la drogue. L'homicide est souvent spontané et non prémédité et les individus vivent rarement dans la même maison. Un élément important rencontré dans plusieurs des histoires de cas est l'état d'intoxication extrême de l'agresseur et/ ou de la victime, altérant ainsi leur réalité. Certains agresseurs plus âgés (40 ans et plus) vivent avec leur victime depuis un certain nombre d'années. Ils sont habituellement tous les deux célibataires et leurs expériences sociales sont très limitées. Le passage à l'acte survient souvent lors d'une al tercation en apparence anodine. Dans certains cas, l'agresseur était violent physiquement depuis une longue période à l'égard de la victime. La seconde catégorie de sujets souffre de pathologie mentale sévère; en effet, dans certains cas, on retrouve des éléments psychotiques tels que des idées délirantes de persécution et de contrôle et/ ou des hallucinations auditives. Souvent, un diagnostic de schizophrénie est présent depuis longtemps. Q uelquefois, dans ce contexte, l'agresseur peut en venir à tuer plus d'une personne.

Q uelques lignes maintenant sur les aspects préventifs. Certains points sont importants chez les enfants. Le premier est d'éviter une absence prolongée des parents du domicile familial ; en effet, certains croient à tort que de jeunes enfants peuvent s'occuper de nouveau-nés. D es mesures sévères doivent aussi être prises afin d'empêcher l'accès aux armes à feu. De plus, les parents devraient mieux préparer les enfants à la venue d'un autre membre dans la famille (naissance ou adoption); certains en viennent à penser qu'il ont mal agi et que leurs parents ne les aiment plus. Ils devraient 
également éviter les comparaisons entre les enfants et leur apprendre à utiliser des techniques de résolution de conflits. Comme le mentionnaient Kashani et al. (1997), les parents doivent être conscients des forces destructives de la jalousie entre frères et/ ou sœurs. En clinique, plusieurs points devraient être vérifiés en entrevue avec les enfants et/ ou les parents: (1) leur colère/ hostilité à l'égard des membres de la famille; (2) leur impulsivité; (3) leur habileté à résoudre des conflits; (4) leur habileté à composer avec des comportements perçus comme provocants; (5) la disponibilité d'armes; (6) et la présence d'un plan précis de passage à l'acte.

Chez les adolescents, les parents ont également un rôle important à jouer. L'apprentissage de techniques de résolution des conflits est à encourager. La tolérance à la frustation/ situation stressante constitue aussi une autre avenue essentielle. De plus, les comportements violents, autant physiques que verbaux, entre membres de la même fratrie ne doivent pas être encouragés, ni le favoritisme, ni le dénigrement systématique de certains membres de la fratrie. Plusieurs souffrent de troubles des conduites (amoralité, absence de culpabilité, etc.) et cet aspect mérite d'être pris en considération, surtout en présence d'une consommation de psychotropes. Patterson (1943), il y a longtemps, suggérait notamment d'être vigilant à la sensation de désespoir combinée à la colère/ frustation qui en résulte chez les adolescents. Les parents et/ ou les professeurs doivent être vigilants lors de l'apparition de ces symptômes. Dans les cas de ruptures d'union et de reconstitutions des familles, il importe de laisser le temps et la place nécessaires à l'adaptation à une nouvelle situation, car cette période est importante pour l'expressivité et la définition d'une identité propre pour plusieurs.

Chez les adultes, plusieurs souffrent d'une pathologie mentale sévère. La plupart des cas recensés dans la littérature montraient des signes évidents d'éléments psychotiques perçus par les membres de la famille. Cependant, plusieurs victimes ont banalisé de nombreux signaux sur une longue période de temps (Roman et Annis, 1986). II est important de rappeler que la présence d'idées délirantes (de persécution ou de contrôle) et/ ou de comportements/ discours désorganisés doit amener une réflexion des apparentés sur la dangerosité de la personne. Pourtant, ils ont souvent tendance à croire qu'ils sont protégés, voire immunisés, contre les comportements violents de l'agresseur. Enfin, les situations où les individus effectuent un passage à l'acte lors d'intoxication sont plutôt difficiles à prévoir, compte tenu de la spontanéité du geste. 
Il est important de mentionner certaines limites à cette synthèse. Les informations dérivées de certaines variables (surtout psychologiques) proviennent de plusieurs auteurs, de disciplines et de traditions de recherche différentes. II est possible que certains auteurs mettent l'accent sur des éléments plutôt que sur d'autres dans leur description du passage à l'acte et que cette coloration soit présente dans la revue des écrits. En outre, les informations recueillies dans les rapports de cas sont tributaires de la connaissance de l'histoire de l'agresseur par les auteurs. Par exemple, on ne sait pas toujours clairement si les auteurs ont bien fait la distinction entre les différents types de fratrie (frères/ sœurs biologiques, adoptés, demi-frères/ sœurs). En dernier lieu, il faut garder à l'esprit que les cas recensés dans cette synthèse ne forment qu'une infime fraction des cas de fratricides/ sororicides commis et que certains éléments psychologiques et criminologiques présents dans des histoires non publiées n'ont pu être rapportés.

Plusieurs thématiques restent à approfondir dans le domaine des fratricides/ sororicides, comme celle du profil des agresseurs de sexe féminin et celle des agresseurs où plusieurs victimes sont en cause. Le rôle de la victime lors du passage à l'acte reste également à déterminer. II devient égal ement important de faire d'autres recherches de nature clinique sur une population adulte. Le peu de références sur les adultes est surprenant dans le contexte où ils forment la majorité des individus effectuant un passage à l'acte dans les enquêtes épidémiologiques.

\section{Références}

Adam, B.S., \& Livingston, R. (1993). Sororicide in preteen girls: a case report and literature review. A cta Paedopsychiatrica, 56, 47-51.

Adelson, L. (1972). The battering child. The Journal of the A merican M edical A ssociation, 222, 159-161.

Benedek, E.P., \& Cornell, D.G. (1989). Clinical presentations of homicidal adolescents. In E.P. Benedek \& D.G. Cornell (eds), Juvenile homicide (40-45). Washington: American Psychiatric Press Inc.

Boisvert, R ., \& Cusson, M. (1999). Homicides et autres violences conjugales. In J. ProulX, M. Cusson \& M. O uimet (eds), Les violences criminelles (77-89). Sainte-Foy : Les Presses de l'U niversité Laval.

Bornstein, S., Guegen, B., \& Hache, E. (1995). Syndrome d'Elpénor ou meurtre somnambulique. Société M édico-P sychologique, 154, 195-201.

B riguet-Lamarre, M. (1969). L'adolescent meurtrier : recherche étiologique. Paris: Edouard Privat Éditeur, Bibliothèque de la psychologie clinique.

Carek, D.J., \& Watson, A.S. (1964). Treatment of a family involved in fratricide. A rchives of $G$ eneral Psychiatry, 11, 533-542. 
Cormier, B.M ., Angliker, C.C.J., Gagné, P.W., \& M arkus, B. (1978). Adolescents who kill a member of the family. In J.M. Eckelaar \& S.M. Katz (eds), Family violence (466-478). Toronto : Butterworth.

Coy, J., \& Steinweg, K. (1996). Peacetime clinical experience with «fratricide»: a veterans hospital experience. M ilitary M edicine, 161, 701-703.

Daly, N ., \& Wilson, M . (1988). H omicide. N ew York : Aldine de Gruyter.

Daly, M., Wilson, M., Salmon, C.A., Hiraiwa-H asegawa, M ., \& Hasegawa, T. (2001). Siblicide and seniority. $H$ omicide Studies, 5, 30-45.

Decker, S.H . (1996). Deviant homicide: a new look at the role of motives and victim-offender relationship. Journal of Research in Crime and D elinquency, 33, 427-449.

Dominey, W.J., \& Blumer, L.S. (1984). Cannibalism of early life stages in fishes. In G. H ausfater \& S. Blaffer H rdy (eds), Infanticide (43-64). N ew York: Aldine Publishing Company.

Duncan, J.W., \& Duncan, G.M. (1971). M urder in the family: a study of some homicidal adolescents. A merican Journal of Psychiatry, 127, 1498-1502.

Ewing, C.P. (1990a). K ids who kill. Lexington, M A : Lexington Books.

Ewing, C.P. (1990b). W hen children kill : the dynamics of juvenilehomicide. Lexington, $M$ A : Lexington Books.

Ewing, C.P. (1997). Fatal families: the dynamics of intrafamilial homicide. Thousand 0 aks: Sage Publications.

Fedorowycz, 0. (1999). L'homicide au Canada - 1998. Juristat, 19, 1-15.

Forbes, L.S., \& M ock, D.W. (1994). Proximate and ultimate determinants of avian brood reproduction. In S. Parmigiani \& F.S. vom Saal (eds), Infanticide and parental care (237-256). USA : Harwood Academic Publishers.

Foster, H.H. (1964). Closed files on juvenile homicide: a case report. Journal of 0 ffender Therapy and Comparative Criminology, 8, 56-60.

Gardiner, M . (1985). Ces enfants voulaient-ils tuer? Paris: Petite Bibliothèque Payot.

Gebo, E. (2002). A contextual exploration of siblicide. Violence and Victims, 17, 157-168.

Gelles, R.J., \& Cornell, C.P. (1990). Intimate violence in families. London: Sage Publications.

Hillbrand, M., Alexandre, J.W., Young, J.L., \& Spitz, R.T. (1999). Parricides: characteristics of offenders and victims, legal factors, and treatment issues. A ggression and Violent Behavior, 4, 179-190.

K ashani, J.H., D arby, P.J., Allan, W.D., H artke, K .L., \& Reid, J.C. (1997). Intrafamilial homicide committed by juveniles: examination of a sample with recommandations for prevention. Journal of Forensic Sciences, 42, 873-878.

La Sainte Bible (1959). Éditions de M aredsous. Braine-le-Comte: Éditions Zech et fils.

Leong, G.B. (1989). Clinicolegal issues for the forensic examiner. In E.P. Benedek \& D.G. Cornell (eds), Juvenile homicide (115-144). Washington : American Psychiatric Press Inc.

Robert, P. (1987). Dictionnaire al phabétique et analogique de la langue française. Paris: Dictionnaire Le Robert. 
Leung, A.K.D., \& Robson, W.L.M . (1991). Sibling rivalry. Clinical Pediatrics, 30, 314-317.

M ahieu, E. (1998). Q uelques considérations sur le fratricide. L'information Psychiatrique, 2, 131-140.

$M$ archand, P., \& Basse, F. (1986). Autour du déterminisme d'un fratricide. Société M édico-P sychologique, 144, 531-534.

M arleau, J.D., \& Saucier, J.-F. (1998). Birth order and fratricidal behavior in Canada. Psychological Reports, 82, 817-818.

M arleau, J.D., Roy, R., Laporte, L., Poulin, B., \& Webanck, T. (1995). H omicide d'enfant commis par la mère. Canadian Journal of Psychiatry, 40, 142-149

M arleau, J.D., Poulin, B., Webanck, T., Roy, R., \& Laporte, L. (1999). Paternal filicide: a study of 10 men. Canadian Journal of Psychiatry, 44, 57-63.

M cCully, R.S. (1978). The laugh of Satan : a study of a familial murderer. Journal of Personality Assessment, 42, 81-91.

M edlicott, R.W. (1970). A study of two fratricides. A maintenance of normal ego functioning in the presence of psychotic ego organisations. Australian and $\mathrm{N}$ ew Z eal and Journal of C riminology, 3, 69-75.

M illaud, F., Auclair, N., \& M eunier, D. (1996). Parricide and mental illness: a study of 12 cases. International Journal of Law and Psychiatry, 19, 173-182.

M illaud, F., Roy, R ., G endron, P., \& Aubut, J. (1992). Un inventaire pour l'évaluation de la dangerosité des patients psychiatriques. Canadian Journal of Psychiatry, 37, 608-615.

M iller, D., \& Looney, J. (1974). The prediction of adolescent homicide: episodic dyscontrol and dehumanization. International Journal of P sychoanalysis, 34 , 187-198.

M ock, D.W. (1984). Infanticide, siblicide, and avian nestling mortality. In G. Hausfater \& S. Blaffer H rdy (eds), Infanticide (3-30). N ew York: Aldine Publishing Company.

0 chonisky, A. (1963). Contribution à l'étude du parricide: à propos de douze observations cliniques. La Psychiatrie del'enfant, 6, 411-487.

Paluszny, M ., \& M cN abb, M . (1975). Therapy of a 6 -year-old who committed fratricide. Journal of the A merican A cademy of Child and A dolescent Psychiatry, 14, 319-336.

Patterson, R.M . (1943). Psychiatric study of juveniles involved in homicide. A merican Journal of Orthopsychiatry, 135, 125-126

Petit, T.A., \& Wells, K. (1980). Crisis treatment of a preadolescent who killed his twin. A merican Journal of Psychotherapy, 34, 434-443.

Polis, G.A. (1984). Intraspecific predation and «infant killing» among invertebrates. In G. H ausfater \& S. B laffer H rdy (eds), Infanticide (87-104). N ew York: Aldine Publishing Company.

Rabain, J.-F. (1995). La rivalité fraternelle. In S. Lebovici, R. Diatkine \& M. Soule (eds), N ouveau traité de psychiatrie de l'enfant et de l'adolescent (25492576), vol IV. Paris: Presses universitaires de France.

Reouven, R. (1986). D ictionnaire des assassins: d'A bimelech à Zulotea, de Caïn à $M$ esrine. Paris: Éditions D enoël. 
Roman, D.D., \& Annis, L.V. (1986). Behavioral and situational clues to a psychiatrist's murder by her brother. International Journal of 0 ffender Therapy and Comparative Criminology, 30, 177-182.

Russell, D.H . (1986). A study of juvenile murderers of family members. International Journal of 0 ffender Therapy and Comparative Criminology, 28, 177-192.

Sadoff, R.L. (1995). M others who kill their children. Psychiatric Annals, 25, 601-605.

Sargent, D. (1962). Children who kill - A family conspiracy. Social W ork, 7, 3542.

Scherrer, P. (1980). Crimes et violences dans la famille. In P. Scherrer (ed.), A pproche de la psychiatrie (458-490), Tome 3. France: SIM EP.

Schmideberg, M. (1973). Juvenile murderers. International Journal of Offender Therapy and Comparative Criminology, 17, 241-245.

Stone, A.A. (1993). M urder with no apparent motive. The Journal of Psychiatry and Law, 21, 175-189.

Sturner, W.Q., Sweeney, K.G., Callery, R.T., \& Haley, N.R. (1991). Cocaine babies: the scourge of the ' $90 \mathrm{~s}$. Journal of Forensic Sciences, 36, 34-39.

Sulloway, F.J. (1996). Born to rebel : birth order, family dynamics, and creative lives. $\mathrm{N}$ ew York: Pantheon Book.

Tooley, K. (1975). The small assassins: clinical notes on a subgroup of murderous children. Journal of the A merican A cademy of Child Psychiatry, 14, 306318.

Underwood, R.C., \& Patch, P.C. (1999). Siblicide: a descriptive analysis of sibling homicide. H omicide Studies, 3, 333-348.

Wallace, A. (1986). H omicide: the social reality. N SW bureau of C rime Statistics and Research/ Attorney General's D epartment.

Williams, B.E. (1988). Conspiracy of silence: staff management crisis on an adolescent milieu unit. The Clinical Supervisor, 6, 83-100.

Woods, S.M . (1961). Adolescent violence and homicide. Archives of $G$ eneral Psychiatry, 5, 528-534.

Woytassek, L.E., \& Atwal, S.S. (1985). Capgras syndrome in court. N ebraska M edical Journal, 70, 392-394.

Zagury, D., \& Millaud, F. (1998). Le passage à l'acte du parricide psychotique. In F. Millaud (ed.), Le passage à l'acte: aspects cliniques et psychodynamiques (119134). Paris: Masson. 